\title{
EMBEDDINGS IN DIVISION RINGS
}

\author{
BY \\ JOHN DAUNS
}

\begin{abstract}
A method for embedding a certain class of integral domains in division rings is devised. Integral domains $A$ are constructed with a generalized valuation into a (noncommutative) totally ordered semigroup that need not be discrete. Then the multiplicative semigroup $A \backslash\{0\}$ is expressed as an inverse limit of semigroups each of which is embeddable in a group. Thus $A \backslash\{0\}$ can be embedded in a group $G$. The main problem is to introduce addition on $G$ in order that $G$ becomes a division ring by the use of eventually commuting maps of inverse limits.
\end{abstract}

Introduction. Suppose that the multiplicative semigroup $A^{*}$ of a noncommutative integral domain $A$ can be embedded in a group $G$. Some recent surprising discoveries show that there exist rings $A$ for which an embedding $A^{*} \subset G$ is possible, but such that for any embedding of $A^{*}$ into any group $G$ whatever, addition cannot be extended to all of $G \cup\{0\}$ in order to obtain an embedding of $A$ into a division ring ([1] and [6]). Under certain appropriate additional hypotheses on an integral domain with a valuation into the integers, P. M. Cohn embeds $A \subset G \cup\{0\}$, introduces a group topology on $G$, then defines addition on the subset $A^{*} A^{*-1}$ which happens to be dense in $G$, and then finally extends addition to all of $G$ [2]. Recent interest in the subject ([1], [6], and [3]), as well as the fact that treatises on ring theory find it necessary to quote this result [5, p. 257] but do not prove it because existing proofs are too complicated, are two reasons why a purely algebraic proof which avoids topology altogether is needed.

The first objective of this note is to introduce a much wider class of rings than simply integral domains with an integer valued valuation. The second aim is to prove that any ring in this wider class can be embedded in a division ring. One of the main factors contributing to the length and complexity of the present proof is that in place of the integers, the range of the valuation is in a not necessarily commutative semigroup $\Gamma$. One of the appealing features of the present development is that if $\Gamma$ is specialized to be commutative, our proof simplifies considerably. Furthermore, it involves no topological considerations of any kind whatsoever.

1. Rings with a noncommutative valuation. In an ordered ring, the valuation

Received by the editors February 24, 1969 and, in revised form, November 25, 1969.

AMS Subject Classifications. Primary 1646, 1615; Secondary 2092.

Key Words and Phrases. Integral domain, division ring, generalized valuation into a semigroup, Ore condition, eventually commuting maps of inverse limits, P. M. Cohn's embedding theorem, totally ordered cancellative semigroup, semigroup congruence.

Copyright (C) 1970, American Mathematical Society 
should be an order preserving map. For this reason unfortunately, the order relation in the subsequent definition is just the reverse of that commonly accepted by most of the previous authors.

1.1 Definition. Consider a totally ordered semigroup $\Gamma$, i.e. $\Gamma$ is a totally ordered set, where if $\alpha \leqq \beta, \bar{\alpha} \leqq \bar{\beta}$, then $\alpha \bar{\alpha} \leqq \beta \bar{\beta}$. Suppose $\Gamma$ is cancellative $(\alpha y=\beta y$ or $y \alpha=y \beta$ implies $\alpha=\beta)$. Let $\Gamma \cup\{0\}$ be the ordered semigroup where $0 \gamma=\gamma 0<\Gamma$ for all $\gamma \in \Gamma$. Here, a ring with a valuation is a triple $A, v, \Gamma$ where $v: A \rightarrow \Gamma \cup\{0\}$ is a function such that for all $a, b \in A$, the following are satisfied

(i) $v(a)=0$ if and only if $a=0$,

(ii) $v(a-b) \leqq \max [v(a), v(b)]=v(a) \vee v(b)$,

(iii) $v(a b)=v(a) v(b)$.

For any semigroup $\Gamma$, as usual $\Gamma^{1}=\Gamma$ if $\Gamma$ has an identity, or $\Gamma^{1}=\Gamma \cup\{1\}$ is $\Gamma$ with an identity adjoined otherwise. Let $P \subseteq \Gamma$ be the strict two-sided increasers, i.e. $P=\{\gamma \in \Gamma \mid y<\gamma y, y<y \gamma$ for all $y \in \Gamma\}$. If $P$ is nonvoid, then it is a subsemigroup.

1.2. The following identities valid for all $a, b \in A^{*}=A \backslash\{0\}$ are proved just the same as in the case when $\Gamma$ are the integers under addition.

(i) $v(-a)=v(a)$,

(ii) $v(b)<v(a) \Rightarrow v(a-b)=v(a)$,

(iii) $v(a) \neq v(b) \Rightarrow v(a-b)=v(a) \vee v(b)$,

(iv) $v(a)>v(a-b) \Rightarrow v(a)=v(b)$,

(v) $A$ is an integral domain.

1.3 Definition. For $\gamma \in P$, let $q(\gamma)$ denote all $(a, b) \in A^{*} \times A^{*}$ such that for any $s \in \Gamma^{1}$, the following two equations hold

(i) $\gamma s v(a-b) \leqq s v(a)$,

(ii) $v(a-b) s \gamma \leqq v(a) s$.

To recapitulate, a product of $\gamma, s$, and $v(a-b)$ is less than $s v(a)$ or $v(a) s$, where $s$ precedes $v(a)$ if and only if it also precedes $v(a-b)$. In subsequent proofs it will automatically be assumed that $s \in \Gamma^{1}$ is an arbitrary element without quantifying $s$ each time.

1.4 REMARK. The following observations will not be used in subsequent proofs. If $\dot{E}=\{\gamma \in \Gamma \mid y<\gamma y$, all $y \in \Gamma\}$ and $R$ are the subsemigroups of strict left and right increasers of $\Gamma$, then define

$$
\begin{array}{ll}
\text { for } \gamma \in L, & q(\gamma, L)=\left\{(a, b) \in A^{*} \times A^{*} \mid \gamma v(a-b) \leqq v(a)\right\} ; \\
\text { for } \gamma \in R, & q(\gamma, R)=\left\{(a, b) \in A^{*} \times A^{*} \mid v(a-b) \gamma \leqq v(a)\right\} ; \\
\text { for } \gamma \in P=L \cap R, & q(\gamma, P)=q(\gamma, L) \cap q(\gamma, R) .
\end{array}
$$

All three are equivalence relations. For an arbitrary $c \in A^{*}$, the following implications hold

$$
\begin{aligned}
& (a, b) \in q(\gamma, L) \Rightarrow(a c, b c) \in q(\gamma, L), \\
& (a, b) \in q(\gamma, R) \Rightarrow(c a, c b) \in q(\gamma, R) .
\end{aligned}
$$

Unfortunately, there is no reason why $(a, b),(c, d) \in q(\gamma, P)$ should imply that 
also $(a c, b d) \in q(\gamma, P)$. Nevertheless, the latter does hold if $\Gamma$ is commutative. In that case we may take $q(\gamma, P)=q(\gamma, L)=q(\gamma, R)$ in place of $q(\gamma)$ in Definition 1.3 and all the subsequent proofs become much simpler. However, one of our main objectives is to embed $A$ in a division ring when $\Gamma$ is not commutative.

1.5 Lemma. If $A, v, \Gamma$ is a valuated ring, then $q(\gamma)$ is an equivalence relation for each $\gamma \in P$.

Proof. Symmetric. If $a=b$, then the left side of 1.3 (i) and (ii) contains $0=v(a-a)$, hence equals 0 , and thus is (strictly) less than the right side.

Reflexive. If $(a, b) \in q(\gamma)$, then $v(a-b)<\gamma v(a-b) \leqq v(a)$. By 1.2 (iv), $v(a)=v(b)$. Replacement of $v(a)$ by $v(b)$ in 1.3 (i), (ii) shows that also $(b, a) \in q(\gamma)$.

Transitive. Given $(a, b)$ and $(b, c) \in q(\gamma)$, then as above $v(a)=v(b)=v(c)$. Thus 1.3 (i) and (ii) become

(i) $\gamma s v(a-c) \leqq \gamma s v(a-b) \vee \gamma s v(c-b) \leqq s v(a)$,

(ii) $v(a-c) s \gamma \leqq v(a-b) s \gamma \vee v(c-b) s \gamma \leqq v(a) s$.

Hence also $(a, c) \in q(\gamma)$.

1.6. For each $\gamma \in P, q(\gamma)$ is a semigroup congruence.

Proof. For $(a, b),(c, d) \in q(\gamma)$, perform the indicated operations, where $s, \bar{s} \in \Gamma^{1}$ are arbitrary:

(i) $\gamma s v(a-b) \leqq s v(a), s=\bar{s} v(c) \Rightarrow \gamma \bar{s} v(c a-c b) \leqq \bar{s} v(c a)$;

(ii) $v(a-b) s \gamma \leqq v(a) s \Rightarrow v(c a-c b) \bar{s} \gamma \leqq v(c a) \bar{s}$.

Thus $(c a, c b) \in q(\gamma)$, and similarly

(i) $\gamma s v(c-d) \leqq s v(c) \Rightarrow \gamma \bar{s} v(c b-d b) \leqq \bar{s} v(c b)$;

(ii) $v(c-d) s \gamma \leqq v(c) s, s=v(b) \bar{s} \Rightarrow v(c b-d b) \bar{s} \gamma \leqq v(c b) \bar{s}$.

Hence $(c b, d b) \in q(\gamma)$. Finally, by transitivity, also $(c a, d b) \in q(\gamma)$.

1.7. For $\gamma \in P$, let $S_{\gamma}=A^{*} / g(\gamma)$. Let $q(\gamma): A^{*} \rightarrow S_{\gamma}$ be the projection given by the equivalence relation $q(\gamma)$, where $a q(\gamma)=\left\{b \in A^{*} \mid(a, b) \in q(\gamma)\right\}$. For $(a, b) \in q(\gamma)$, $a q(\gamma)=b q(\gamma) \in S_{\gamma}$ and abbreviate this by $a \sim b \bmod (\gamma)$. If $\alpha<\beta$ with $\alpha \in P$, then $q(\beta) \subseteq q(\alpha)$ and thus there is an induced map $\varphi_{\alpha}^{\beta}: S_{\beta} \rightarrow S_{\alpha}$, where $a q(\beta) \varphi_{\alpha}^{\beta}=a q(\alpha)$. Thus $\left\{\varphi_{\alpha}^{\beta}: S_{\beta} \rightarrow S_{\alpha} ; \alpha<\beta ; \alpha, \beta \in P\right\}$ is an inverse system of semigroups with inverse limit $S$ and maps $S \rightarrow S_{\alpha}$ for each $\alpha \in P$ (for definition of $S$ and $S \rightarrow S_{\alpha}$, see $\S 2$ ).

1.8. At this point an additional hypothesis on $\Gamma$ and one on $A$ have to be invoked.

(i) In addition to $\Gamma$ being totally ordered and cancellative, assume that for any $\lambda_{1}, \lambda_{2} \in \Gamma$ with $\lambda_{1} \leqq \lambda_{2}$, there is a $\gamma \in P$ such that $\lambda_{2}<\gamma \lambda_{1}$.

(ii) In addition to $A$ being a valuated integral domain, assume that for any $\gamma \in P$ and any $a, b \in A^{*}$, there exist $r, \bar{r} \in A^{*}$ such that $(a r, b \bar{r}) \in q(\gamma)$. Furthermore, assume that the cancellativity condition that for $c \in A^{*}$, if either $(a c, b c) \in q(\gamma)$ or $(c a, c b) \in q(\gamma)$, that then also $(a, b) \in q(\gamma)$.

1.9 Remarks (1) Note that condition 1.8 (ii) implies that $S_{y}$ may be embedded in a group, i.e. that $S_{\gamma}$ is cancellative on both sides and it satisfies the so-called right Ore condition that for any $\gamma \in P$ and any $x, y \in S_{\gamma}, x S_{\gamma} \cap y S_{\gamma} \neq \varnothing$. 
(2) If $\Gamma$ is commutative, then the cancellativity requirement in 1.8 (ii) is superfluous, since then it follows immediately from 1.3 and the fact that $\Gamma$ is cancellative.

(3) The whole theory could equally well be developed by imposing analogous conditions in both (i) and (ii) on the other (right) side.

1.10. If $(a, b) \in \bigcap\{q(\gamma) \mid \gamma \in P\}$ then in particular $\gamma v(a-b) \leqq v(a)$ holds for all $\gamma \in P$. But for $a \neq b$, if $\lambda_{1}=v(a-b) \neq 0, \lambda_{2}=v(a)$ and $\gamma$ is as in 1.8 (i), then $v(a)$ $<\gamma v(a-b)$. Thus $\bigcap\{q(\gamma) \mid \gamma \in P\}=\left\{(a, a) \mid a \in A^{*}\right\}$, and the semigroup homomorphisms $q(\beta): A^{*} \rightarrow S_{\beta}$ which commute with the $\varphi_{\alpha}^{\beta}$ induce an isomorphism $q: A^{*} \rightarrow S$. By cancellativity and the right Ore condition 1.8 (ii), each $S_{\beta}$ may be embedded in its group of quotients $G_{\beta}$. That is, for $x, y \in S_{\beta}$, let

$$
x / y=\left\{(s, t) \in S_{\beta} \times S_{\beta} \mid x r=s \bar{r}, y r=t \bar{r} \text { for some } r, \bar{r} \in S_{\beta}\right\}
$$

and define a group operation on the set $G_{\beta}=\left\{x / y \mid x, y \in S_{\beta}\right\}$ by $(x / y)(u / w)=x \rho / w \bar{\rho}$, where $y \rho=u \bar{\rho} \in y S_{\beta} \cap u S_{\beta}$. If $e \in S_{\beta}$ is arbitrary, then the embedding $S_{\beta} \cong$ $\left\{s e / e \mid s \in S_{\beta}\right\} \subset G_{\beta}$ is independent of $e$. Any homomorphism $\varphi_{\alpha}^{\beta}: S_{\beta} \rightarrow S_{\alpha}$ into any other cancellative semigroup $S_{\alpha}$ also with a right Ore condition, extends uniquely to a homomorphism $\pi_{\alpha}^{\beta}: G_{\beta} \rightarrow G_{\alpha}$ of their respective groups of quotients, where $(x / y) \pi_{\alpha}^{\beta}=x \varphi_{\alpha}^{\beta} / y \varphi_{\alpha}^{\beta}$. Then $\left\{\pi_{\alpha}^{\beta}: G_{\beta} \rightarrow G_{\alpha} ; \alpha<\beta ; \alpha, \beta \in P\right\}$ is an inverse system of groups with an inverse limit $G$ and the usual maps $G \rightarrow G_{\alpha}$ for $\alpha \in P$. Furthermore, a monomorphism $S_{\beta} \rightarrow G_{\beta}, \beta \in P$, of inverse systems always induces also a semigroup monomorphism $S \rightarrow G$. Abbreviate the element $a q(\beta) / b q(\beta)$ of $G_{\beta}$ simply as $a / b \bar{q}(\beta)$, and the equality $a / b \bar{q}(\beta)=c / d \bar{q}(\beta)$ by $a / b \equiv c / d \bmod (\beta)$. Thus equality in $G_{\beta}$ will be denoted by "三” and in $S_{\beta}$ by " $\sim$ ". There are the usual commutative diagrams for $\alpha<\beta \in \Gamma$.
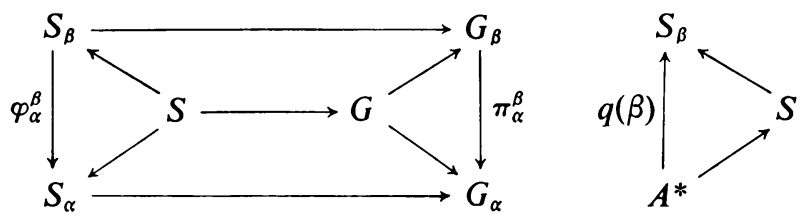

Note that in the next definition if $\Gamma$ is commutative, $\delta \triangleleft \alpha$ means simply that $\delta<\alpha$.

1.11 Definition. For $\delta, \alpha \in P$ call $\delta$ much less than $\alpha$ and write $\delta \triangleleft \alpha$ provided the following two conditions hold for any $\lambda_{1}, \lambda_{2}, \mu \in \Gamma$. Any one of the three inequalities on the left imply that all three on the right hold both in (i) and (ii).

(i) $\alpha \lambda_{1} \lambda_{2} \leqq \mu$, or $\lambda_{1} \alpha \lambda_{2} \leqq \mu$, or $\lambda_{1} \lambda_{2} \alpha \leqq \mu \Rightarrow \delta \lambda_{1} \lambda_{2}<\mu, \lambda_{1} \delta \lambda_{2}<\mu, \lambda_{1} \lambda_{2} \delta<\mu$;

(ii) $\mu \leqq \delta \lambda_{1} \lambda_{2}$, or $\mu \leqq \lambda_{1} \delta \lambda_{2}$, or $\mu \leqq \lambda_{1} \lambda_{2} \delta \Rightarrow \mu<\alpha \lambda_{1} \lambda_{2}, \mu<\lambda_{1} \alpha \lambda_{2}, \mu<\lambda_{1} \lambda_{2} \alpha$.

The main objective now is to introduce subtraction in $G$ compatible with the embedding $A \subset G \cup\{0\}$.

1.12. Suppose $\delta, \alpha, \beta$ are any three elements of $P$ satisfying $\delta \triangleleft \alpha<\alpha^{2} \leqq \beta$. 
Define $Y \subset G_{\beta}$ as the set of all $x \in G_{\beta}$ such that no matter which representatives $a, b \in A^{*}$ are chosen which give $x=a / b \bar{q}(\beta)$, they satisfy $v(a) \vee v(b) \leqq \delta v(a \cdot-b)$. Define $\psi_{\alpha}^{\beta}: Y \rightarrow G_{\alpha}$ by $x \psi_{\alpha}^{\beta}=(b-a) / b \bar{q}(\alpha)$. It has to be shown that $\psi_{\alpha}^{\beta}$ is well defined. Suppose that $x$ is also represented as $x=a_{1} / b_{1} \bar{q}(\beta)$. This implies that $a r \sim a_{1} \bar{r}$ and $b r \sim b_{1} \bar{r} \bmod (\beta)$ for $r, \bar{r} \in A^{*}$. It suffices to show that $(b-a) r \sim\left(b_{1}-a_{1}\right) \bar{r} \bmod (\alpha)$. But this is a consequence of the next lemma (with $k=b r, m=a r, k_{1}=b_{1} \bar{r}$, and $\left.m_{1}=a_{1} \bar{r}\right)$.

1.13 Lemma. Consider $\delta \triangleleft \alpha<\alpha^{2} \leqq \beta$, where $\delta \in P$ and $k, k_{1}, m, m_{1} \in A^{*}$ with $k \sim k_{1}$ and $m \sim m_{1} \bmod (\beta)$, and $v(k) \vee v(m) \leqq \delta v(k-m)$. Then $k-m \sim k_{1}-m_{1}$ $\bmod (\alpha)$

Proof. For any $s \in \Gamma^{1}$, straightforward estimates show that

$$
\begin{aligned}
\beta s v\left(k-m-k_{1}+m_{1}\right) & \leqq \beta s v\left(k-k_{1}\right) \vee \beta s v\left(m-m_{1}\right) \\
& \leqq s v(k) \vee s v(m)=s[v(k) \vee v(m)] \\
& \leqq s \delta v(k-m)<\alpha s v(k-m) .
\end{aligned}
$$

Use of $\alpha^{2} \leqq \beta$ and cancellation of $\alpha$ gives 1.3 (i) while an entirely parallel argument establishes 1.3 (ii). Thus $k-m \sim k_{1}-m_{1} \bmod (\alpha)$.

1.14 Remark. In the context of 1.12 , define $Z$ as the set $Y \subseteq Z \subset G_{\beta}$ consisting of all $x \in G_{\beta}$ such that there exist some representatives $a, b \in A^{*}$ with $x=a / b \bar{q}(\beta)$ such that $v(a) \vee v(b) \leqq \delta v(a-b)$. Suppose also $x=a_{1} / b_{1} \bar{q}(\beta)$, where it is not assumed that $a_{1}, b_{1}$ satisfy the last inequality. As before let $a r \sim a_{1} \bar{r}$ and $b r \sim b_{1} \bar{r} \bmod (\beta)$ for $r, \bar{r} \in A^{*}$. Then the above arguments actually show that $(b-a) r \sim\left(b_{1}-a_{1}\right) \bar{r}$ $\bmod (\alpha)$; hence $(b-a) / b \equiv\left(b_{1}-a_{1}\right) / b_{1} \bmod (\alpha)$. Thus the domain of the map $\psi_{\alpha}^{\beta}$ could be enlarged to $Z$.

Some final restrictions in addition to 1.8 (i) and (ii) have to be imposed on $\Gamma$.

1.15. Assume that there is a cofinal subset $P_{1}$ in $P_{1} \subseteq P$ and two order preserving maps $\theta: P_{1} \rightarrow P$, and $\sigma: P_{1} \rightarrow P$ satisfying:

(i) $\sigma(\beta) \triangleleft \theta(\beta)<\theta(\beta)^{2} \leqq \beta$ for all $\beta \in P_{1}$.

(ii) For any $\eta \in \Gamma$, there exist $\alpha_{1}, \alpha_{2} \in P_{1}$ with $\eta<\sigma\left(\alpha_{1}\right) \triangleleft \theta\left(\alpha_{1}\right) \leqq \alpha_{1}<\sigma\left(\alpha_{2}\right)$ $\triangleleft \theta\left(\alpha_{2}\right) \leqq \alpha_{2}$.

1.16 Remarks. 1 . Note that 1.15 (ii) implies that both $\theta\left(P_{1}\right)$ and $\sigma\left(P_{1}\right)$ are cofinal in $P$, that $P$ is cofinal in $\Gamma$, and that $\sigma$ and $\theta$ are order decreasing maps.

2. Actually 1.15 (ii) is equivalent to (ii') below, which at first glance appears to be a weaker assumption.

(ii') For any $\eta \in \Gamma$, there exists $\alpha \in P_{1}$ with $\eta<\sigma(\alpha)$.

3. In the examples of totally ordered semigroups $\Gamma$ given at the end, $P \neq \varnothing$ and each $\alpha \in P$ satisfies $\alpha \triangleleft \alpha^{2}$. In this case there is a natural choice of $P_{1}, \theta$, and $\sigma$ satisfying the conditions in 1.15. Set $P_{1}=\left\{\alpha^{4} \mid \alpha \in P\right\}, \theta\left(\alpha^{4}\right)=\alpha^{2}$, and $\sigma\left(\alpha^{4}\right)=\theta\left(\theta\left(\alpha^{4}\right)\right)$ $=\alpha$. Then 1.15 (i) and (ii) clearly hold.

The next definition combines 1.12 with 1.15 . If $\eta>\delta \in P$, then $\eta \in P$. Thus if $P \neq \varnothing, P$ is cofinal. 
1.17 Definition. For $\Gamma$ as in 1.8 and 1.15 and for any $\beta \in P_{1}$, define $Y_{\beta} \subseteq G_{\beta}$ as all $x \in G_{\beta}$ such that no matter which representatives $a, b \in A^{*}$ of $x=a / b \bar{q}(\beta)$ are chosen, they satisfy the inequality $v(a) \vee v(b) \leqq \sigma(\beta) v(a-b)$. Let $\psi^{\beta}: Y_{\beta} \rightarrow G_{\theta(\beta)}$ be the map $\psi_{\alpha}^{\beta}$ of 1.12 with $\delta=\sigma(\beta) \triangleleft \alpha=\theta(\beta)<\theta(\beta)^{2} \leqq \beta$.

1.18 Lemma. For any $1 \neq x=\left\{x_{\alpha}\right\} \in G$, there is an $\eta=\eta(x) \in P$ such that $x_{\beta} \in Y_{\beta}$ for all $\beta>\eta$.

Proof. Let $1 \neq x_{\mu} \in G_{\mu}$. By 1.15 (ii), choose an element $\eta$ such that $\delta=\sigma(\eta) \in P$ with $\mu \triangleleft \delta$. Suppose an arbitrary $\beta>\eta$ is given. Then $\sigma(\beta)>\sigma(\eta)$. Take any $a, b \in A^{*}$ with $a / b \bar{q}(\beta)=x_{\beta}$. Then $a / b \bar{q}(\mu) \neq 1$ implies $a q(\mu) \neq b q(\mu)$, and thus at least one of the two equations for the equality of $a$ and $b$ modulo $q(\mu)$ in $S_{\mu}$ is violated, i.e. for some $s \in \Gamma^{1}$ ( $s$ depending on $a$ and $b$ ) either

(i) $s v(a)<\mu s v(a-b)<s \delta v(a-b)$, or

(ii) $v(a) s<v(a-b) s \mu<\delta v(a-b) s$.

Thus in either case (i) or (ii), it follows that $v(a)<\delta v(a-b)$. Suppose $\delta v(a-b)$ $<v(b)$ so that $v(a-b)<v(b)$. Then $v(a)=v(b)$ by 1.2 (iv), which contradicts $v(a)$ $<\delta v(a-b)<v(b)$. Thus $v(a) \vee v(b) \leqq \delta v(a-b)$ and $x_{\beta} \in Y_{\beta}$ for all $\beta>\eta$.

2. Inverse limits and eventually commuting maps. The maps $\psi^{\beta}$ for $\beta \in P_{1}$ of the previous section will induce a function $\psi: G \backslash\{1\} \rightarrow G \mid\{1\}$ satisfying the four requirements (see 3.1) for defining subtraction on $G \cup\{0\}$. In order not to obscure the fact that the method for constructing $\psi$ is very generally applicable, and not at all dependent on the various specialized properties of $\Gamma, A$ and the $G_{\alpha}$, this section is developed for an inverse limit of arbitrary sets $X_{\alpha}$ in place of the $G_{\alpha}$ and any arbitrary partially ordered upper directed set $P$.

2.1 Notation. Consider any inverse system of sets $\left\{\pi_{\alpha}^{\beta}: X_{\beta} \rightarrow X_{\alpha} ; \alpha<\beta\right.$; $\alpha, \beta \in P\}$, where $P$ is any upper directed partially ordered set, and where for $\alpha<\beta<\gamma$, the usual identities $\pi_{\beta}^{\gamma} \pi_{\alpha}^{\beta}=\pi_{\alpha}^{\gamma}$ and $\pi_{\alpha}^{\alpha}=1$ are satisfied. Form the inverse limit

$$
X=\lim X_{\alpha} \subset \prod\left\{X_{\alpha} \mid \alpha \in P\right\}, \quad x=\left\{x_{\alpha}\right\} \in X
$$

if and only if for any $\alpha, \beta \in P$ with $\alpha<\beta, x_{\alpha}=x_{\beta} \pi_{\alpha}^{\beta}$.

2.2 Definition. Suppose $P_{1} \subseteq P$ is a subset, and $\theta: P_{1} \rightarrow P$ a given function. Also suppose that for each $\beta \in P_{1}$, there is a subset $Y_{\beta} \subseteq X_{\beta}$ and a function $\psi^{\beta}:-Y_{\beta} \rightarrow X_{\theta(\beta)}$. These $\left\{\psi^{\beta} \mid \beta \in P_{1}\right\}$ are said to commute eventually provided the next three conditions are satisfied.

(i) For any $x=\left\{x_{\alpha} \mid \alpha \in P\right\} \in X$, there is an $\eta(x) \in P$ such that for any $\gamma \in P$ with $\gamma>\eta(x), x_{y} \in Y_{\gamma}$.

(ii) $\theta: P_{1} \rightarrow P$ is an order preserving and order decreasing map (i.e. $\alpha<\beta \Rightarrow \theta(\alpha)$ $\leqq \theta(\beta)$, and $\theta(\alpha) \leqq \alpha)$. For any $\eta \in \Gamma$, there exist $\alpha_{1}, \alpha_{2} \in P_{1}$ with $\eta<\theta\left(\alpha_{1}\right) \leqq \alpha_{1}$ $<\theta\left(\alpha_{2}\right) \leqq \alpha_{2}$.

(Thus both $P_{1}$ and $\theta\left(P_{1}\right)$ are cofinal in $P$.) 
(iii) For any $x \in X$ and any $\delta, \lambda \in P_{1}$ with $\eta(x)<\delta<\lambda$ (and hence also $\theta(\delta)$ $\leqq \theta(\lambda)$ ), the identity holds

$$
x_{\lambda} \pi_{\partial}^{\lambda} \psi^{\delta}=x_{\lambda} \psi^{\lambda} \pi_{\theta(\delta)}^{\theta(\lambda)} \text {. }
$$

2.3 Remark. The conditions in 2.2 say that the diagram

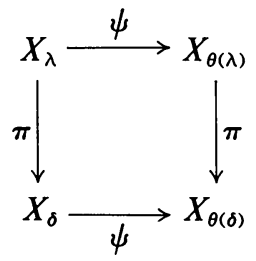

eventually commutes in the sense that for a fixed $x=\left\{x_{\alpha}\right\} \in X$ and for all sufficiently large $\lambda$ with $\delta<\lambda$, the above maps commute pointwise at $x_{\lambda}$.

2.4. Let $\alpha \in P$ and $x=\left\{x_{\alpha}\right\} \in X$ be arbitrary. Define $\psi: X \rightarrow X$ by $x \psi=\left\{x_{\alpha}\right\} \psi$ $=\left\{x_{\alpha}^{\prime}\right\}$, where $x_{\alpha}^{\prime}$ is defined by selecting any $\beta$ with $\beta>\eta(x)$ such that $\theta(\beta)>\alpha$, and then setting $x_{\alpha}^{\prime}=x_{\beta} \psi^{\beta} \pi_{\alpha}^{\theta(\beta)}$. Suppose another element $\gamma$ also satisfying $\gamma>\eta(x)$ with $\theta(\gamma)>\alpha$ had been chosen in place of $\beta$. Set $\tilde{x}_{\alpha}=x_{\gamma} \psi_{1}^{\gamma} \pi_{\alpha}^{\theta(\gamma)}$. In order to show that $x_{\alpha}^{\prime}=\tilde{x}_{\alpha}$, pick any $\lambda$ with $\lambda>\beta, \gamma$, use 2.2(iii) with $\delta=\beta, \gamma$, and then multiply the equation 2.2 (iii) respectively by $\pi_{\alpha}^{\theta(\beta)}, \pi_{\alpha}^{\theta(\gamma)}$ to get

$$
\begin{aligned}
& \delta=\beta: x_{\alpha}^{\prime}=\left(x_{\lambda} \pi_{\beta}^{\lambda} \psi^{\beta}\right) \pi_{\alpha}^{\theta(\beta)}=\left(x_{\lambda} \psi^{\lambda} \pi_{\theta(\beta)}^{\theta(\lambda)}\right) \pi_{\alpha}^{\theta(\beta)}, \\
& \delta=\gamma: \tilde{x}_{\alpha}=\left(x_{\lambda} \pi_{\gamma}^{\lambda} \psi^{\gamma}\right) \pi_{\alpha}^{\theta(\gamma)}=\left(x_{\lambda} \psi^{\lambda} \pi_{\theta(\gamma)}^{\theta(\lambda)}\right) \pi_{\alpha}^{\theta(\gamma)} .
\end{aligned}
$$

Thus $x_{\alpha}^{\prime}=\tilde{x}_{\alpha}$ and $\psi$ is well defined. For later purposes it will be useful to observe that for any $\beta>\eta(x),(x \psi)_{\theta(\beta)}=x_{\theta(\beta)}^{\prime}=x_{\beta} \psi^{\beta}$.

Algebraic properties of eventually commuting maps, such as the idempotence of the map $a / b \rightarrow(b-a) / b$ encountered in the last section, carries over to their inverse limit.

2.5. Assume that the maps $\psi^{\alpha}$ are idempotent in the sense that

$$
x_{\beta} \psi^{\beta} \pi_{\gamma}^{\theta(\beta)} \psi^{\gamma}=x_{\theta(\gamma)}
$$

holds for any $\gamma, \beta$ with $\eta(x)<\gamma<\theta(\beta) \leqq \beta$ (and $\left.\beta, \gamma \in P_{1}\right)$. Then $\psi^{2}=\psi$.

Proof. Define $x^{\prime}=x \psi, x^{\prime \prime}=x^{\prime} \psi$. Now for any $\alpha \in P$ and any $\gamma, \beta \in P_{1}$ sufficiently large, i.e. $\eta(x) \vee \eta\left(x^{\prime}\right) \vee \alpha<\theta(\gamma) \leqq \gamma<\theta(\beta) \leqq \beta$, we have

$$
x_{\alpha}^{\prime \prime}=x_{\gamma}^{\prime} \psi^{\gamma} \pi_{\alpha}^{\theta(\gamma)} \text { and } x_{\gamma}^{\prime}=x_{\beta} \psi^{\beta} \pi_{\gamma}^{\theta(\beta)} .
$$

Thus use of the hypothesis shows that $x_{\alpha}^{\prime \prime}=x_{\theta(\gamma)} \pi_{\alpha}^{\theta(\gamma)}=x_{\alpha}$. Thus $(x \psi) \psi=x$.

2.6 Remark. Since $P_{1}$ is cofinal in $P$, the reader may wish to take the direct limit with respect to $P_{1}$ in place of $P$ in the above considerations.

3. Additive structure on $G \cup\{0\}$. The hypotheses and notation of $\S 1$ are resumed and these are now combined with the results of $\S 2$. That is, $A$ is an integral 
domain with a valuation $v: A \mid\{0\} \rightarrow \Gamma$ into a totally ordered cancellative semigroup $\Gamma$ such that 1.8 (i), (ii) and 1.15 (i), (ii) hold.

The following well-known lemma ([2, p. 514] or $[5$, p. 258]) will be used to introduce subtraction on $G \cup\{0\}$.

3.1 Lemma. Let $G$ be a group and $G_{1}$ the subset $G_{1}=\{x \in G \mid x \neq 1\}$. A necessary and sufficient condition for addition to be definable on $G \cup\{0\}$ so that $G \cup\{0\}$ becomes a division ring with the original group operation of $G$ as multiplication is that there exists an element $e \in G$ and a function $\psi: G_{1} \rightarrow G$ which for any $x, y \in G_{1}$ satisfy the following:

I. $x \psi \psi=x$,

II. $x^{-1} \psi=e(x \psi) x^{-1}$,

III. $\left(y x y^{-1}\right) \psi=y(x \psi) y^{-1}$,

IV. $\left(x y^{-1}\right) \psi\left(y^{-1} \psi\right)^{-1}=\left[(x \psi)(y \psi)^{-1}\right] \psi$ for $x \neq y$.

If there do exist such an $e$ and $\psi$, then $e=-1,-x=e x, x \psi=1-x$, and $x-y$ $=\left[(x \psi)(y \psi)^{-1}\right] \psi(y \psi)=\left(x y^{-1}\right) \psi e y$.

3.2. By 2.5 , the condition $\mathrm{I}$ in 3.1 holds. In II, even if $1 \notin A$, take any $0 \neq c \in A$, and then define $e=\{(-c) / c \bar{q}(\alpha) \mid \alpha \in P\} \in G$. The proof of II is omitted. Verification of any identity, such as II, is usually a routine computation with direct limits, provided the right Ore condition need not be used. However, III and IV do require the Ore condition. In subsequent computations, it will be sometimes convenient to write simply $a / b \in G_{\beta}$ for $a, b \in A^{*}$ in place of the longer expression $a / b \bar{q}(\beta)$.

3.3. In order to prove 3.1 III that $\left(y x y^{-1}\right) \psi=y(x \psi) y^{-1}$, first, it is readily seen that it suffices to show that for $x, y \in G \mid\{1\}$ and for all sufficiently large $\beta$ in $P_{1}$ (for $\eta(y), \eta\left(y^{-1}\right)<\beta$ ) with $x_{\beta}=a / b, y_{\beta}=c / d \in G_{\beta}$, the following holds

$$
c d^{-1}\left(a b^{-1} \psi^{\beta}\right) d c^{-1} \equiv\left(c d^{-1} a b^{-1} d c^{-1}\right) \psi^{\beta} \bmod (\theta(\beta)) .
$$

For any $c, k, n \in A^{*}$,

$$
c\left(k n^{-1} \psi^{\beta}\right) c^{-1} \equiv c \frac{n-k}{n} c^{-1} \equiv \frac{c n-c k}{c n} \equiv \frac{c k}{c n} \psi^{\beta} \equiv\left(c k n^{-1} c^{-1}\right) \psi^{\beta} \bmod (\theta(\beta)) .
$$

That is, (1) holds rather trivially if $d=1$ while (1) with $c=1$ is not so immediate on account of the nonsymmetry in the Ore condition, i.e. $d^{-1}(k / n) d \not \equiv(k d) /(n d)$. It suffices to prove equation (1) with $c=1$, i.e. that

$$
d^{-1}\left(a b^{-1} \psi^{\beta}\right) d \equiv\left(d^{-1} a b^{-1} d\right) \psi^{\beta} \bmod (\theta(\beta))
$$

Assuming (2), we complete the proof by showing (1), and then at the end prove (2). First, since $d^{-1} a b^{-1} d \bar{q}(\beta) \in G_{\beta}$, there are $k$ and $n \in A^{*}$ such that $d^{-1} a b^{-1} d$ $\equiv k n^{-1} \bmod (\beta)$. It now follows from, first, (2) and then (1) that

$$
\begin{aligned}
c\left[d^{-1}\left(a b^{-1} \psi^{\beta}\right) d\right] c^{-1} & \equiv c\left[\left(d^{-1} a b^{-1} d\right) \psi^{\beta}\right] c^{-1} \\
& \equiv c\left[\left(k n^{-1}\right) \psi^{\beta}\right] c^{-1} \equiv\left(c k n^{-1} c^{-1}\right) \psi^{\beta} \\
& \equiv\left(c d^{-1} a b^{-1} d c^{-1}\right) \psi^{\beta} \bmod (\theta(\beta))
\end{aligned}
$$


3.4. To prove (2) above it will be more convenient to show that

$$
d\left[\left(d^{-1} a b^{-1} d\right) \psi^{\beta}\right] d^{-1} \equiv\left(a b^{-1}\right) \psi^{\beta} \bmod (\theta(\beta)) .
$$

Write $b r \sim d \bar{r}, d \rho \sim \operatorname{ar} \bar{\rho} \bmod (\beta)$ for $r, \bar{r}, \rho, \bar{\rho} \in A^{*}$. Then

$$
d^{-1} a b^{-1} d \equiv \frac{1}{d} \frac{a}{b} d \equiv \frac{1}{d} \frac{a r}{b r} \frac{d \bar{r}}{\bar{r}} \equiv \frac{\rho}{d \rho} \frac{a r \bar{\rho}}{\bar{r} \bar{\rho}} \equiv \frac{\rho}{\bar{r} \bar{\rho}} \bmod (\theta(\beta)) .
$$

Use of the latter shows that

$$
d\left[\left(d^{-1} a b^{-1} d\right) \psi^{\beta}\right] d^{-1} \equiv d \frac{\bar{r} \bar{\rho}-\rho}{\bar{r} \bar{\rho}} \frac{1}{d} \equiv \frac{d \bar{r} \bar{\rho}-d \rho}{d \bar{r} \bar{\rho}} \bmod (\theta(\beta)) .
$$

Set $k=b r \bar{\rho} \sim k_{1}=d \bar{r} \bar{\rho}$, and $m=a r \bar{\rho} \sim m_{1}=d \rho \bmod (\beta)$. From the definition of equality in $G_{\beta}$, it follows that $x_{\beta}=a / b \bar{q}(\beta)=m / k \bar{q}(\beta)$. The assumption that $\beta>\eta(x)$ and hence that $x_{\beta} \in Y_{\beta}$ means that for any representatives of $x_{\beta}$, and hence in particular for $m$ and $k$, the inequality $v(k) \vee v(m) \leqq \sigma(\beta) v(k-m)$ holds. Lemma 1.13 with $\alpha=\theta(\beta)$ shows that $k-m \sim k_{1}-m_{1} \bmod (\theta(\beta))$. Since also $k \sim k_{1} \bmod (\beta)$, it follows that $(k-m) / k \equiv\left(k_{1}-m_{1}\right) / k_{1} \bmod (\theta(\beta))$, or equivalently that the following substitutions are legal in

$$
\frac{d \bar{r} \bar{\rho}-d \rho}{d \bar{r} \bar{\rho}} \equiv \frac{b r \bar{\rho}-a r \bar{\rho}}{b r \bar{\rho}} \equiv \frac{b-a}{b} \bmod (\theta(\beta)) .
$$

Thus equation (3) above or equivalently conclusion 3.1 III that $\left(x y x^{-1}\right) \psi=$ $x(y \psi) x^{-1}$ now follows.

3.5. It only now remains to show that for any $x \neq y \in G$ and any $\alpha \in \Gamma$, the $\alpha$ th

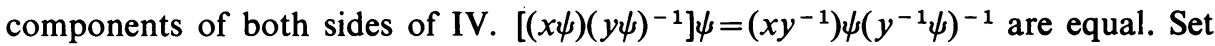
$z=(x \psi)(y \psi)^{-1}$. Then $z \neq 1$, for if $x \psi=y \psi$, then it follows from 2.5 that $x=x \psi^{2}=$ $y \psi^{2}=y$. In order to guarantee that various maps $\psi^{\beta}, \psi^{\gamma}$ can be applied, pick any $\gamma, \beta$ sufficiently large, that is to be precise

$$
\eta(x), \eta(y), \eta\left(x y^{-1}\right), \eta\left(y^{-1}\right), \eta\left((x \psi)(y \psi)^{-1}\right)<\alpha<\theta(\beta) \leqq \beta<\theta(\gamma) \leqq \gamma .
$$

In order to show the equality of the $\alpha$ th component, it merely suffices to show that any higher components, such as $\theta(\beta)$, are equal. Then $(z \psi)_{\theta(\beta)}=z_{\beta} \psi^{\beta}$, where $z_{\beta}$ $=\left(x_{\gamma} \psi^{\gamma}\right)\left(y_{\gamma} \psi^{\gamma}\right)^{-1} \pi_{\beta}^{\theta(\gamma)}$. Thus it suffices to show that

$$
\begin{aligned}
{\left[\left(x y^{-1}\right) \psi\left(y^{-1} \psi\right)^{-1}\right]_{\theta(\beta)} } & =\left(x_{\gamma} y_{\lambda}^{-1}\right) \psi^{\gamma}\left(y_{\gamma}^{-1} \psi^{\gamma}\right)^{-1} \pi_{\theta(\beta)}^{\theta(\gamma)}=z_{\beta} \psi^{\beta} \\
& =\left(x_{\gamma} \psi^{\gamma}\right)\left(y_{\gamma} \psi^{\gamma}\right)^{-1} \pi_{\beta}^{\theta(\gamma)} \psi^{\beta} .
\end{aligned}
$$

Let $x=x_{1} / x_{2}, y=y_{1} / y_{2} \in Y_{y}$ with $x_{1}, x_{2}, y_{1}, y_{2} \in A^{*}$. For any $\zeta \geqq \gamma$, elements $r$, $\bar{r} \in A^{*}$ such that $x_{2} r \sim y_{2} \bar{r} \bmod (\zeta)$ may be chosen and later a judicious choice of $\zeta$ will have to be made. Thus $\left(x y^{-1}\right)_{\gamma} \equiv x_{1} r / y_{1} \bar{r} \in Y_{y}$ also. A straightforward computation shows that the left side of the above equation is

$$
\left(\left(x y^{-1}\right) \psi^{\beta}\right)\left(y^{-1} \psi^{\beta}\right)^{-1} \equiv \frac{x_{1} r-y_{1} \bar{r}}{\left(y_{2}-y_{1}\right) \bar{r}} \bmod (\theta(\beta)),
$$


while the right side becomes

$$
\left(x_{\gamma} \psi^{\gamma}\right)\left(y_{\gamma} \psi^{\gamma}\right)^{-1} \pi_{\beta}^{\theta(\gamma)} \psi^{\beta} \equiv \frac{\left(x_{1} r-y_{1} \bar{r}\right)-\left(x_{2} r-y_{2} \bar{r}\right)}{\left(y_{2}-y_{1}\right) \bar{r}} \bmod (\theta(\beta)) .
$$

Since $x_{2} r \sim y_{2} ; \vec{r} \bmod (\gamma)$, on first glance the reader might simply cancel $x_{2} r-y_{2} \bar{r}$. However, a moments reflection will show that even $v\left(x_{2} r-y_{2} \bar{r}\right)$ might very well dominate the value of any other term in the expression.

3.6. It has now to be shown that for any $s \in \Gamma^{1}$, the usual two inequalities 1.3 (i) and (ii) with $a=x_{1} r-y_{1} \bar{r}$ and $b=a-\left(x_{2} r-y_{2} \bar{r}\right)$ hold:

(i) $\theta(\beta) s v\left(x_{2} r-y_{2} \bar{r}\right) \leqq s v\left(x_{1} r-y_{1} \bar{r}\right)$,

(ii) $v\left(x_{2} r-y_{2} \bar{r}\right) s \theta(\beta) \leqq v\left(x_{1} r-y_{1} \bar{r}\right) s$.

By 1.8 (i) choose $\bar{\lambda} \in P$ such that $v\left(x_{2}\right)<\bar{\lambda} v\left(x_{1}\right)$. Secondly, by 1.15 (ii) choose $\lambda \in P$ such that $\bar{\lambda} \sigma(\gamma) \triangleleft \lambda$; and, thirdly, choose any $\zeta$ satisfying $\theta(\beta) \lambda \vee \lambda \theta(\beta) \leqq \zeta$. Then $x_{2} r \sim y_{2} \bar{r} \bmod (\zeta)$ means that

(l) $v\left(x_{2} r-y_{2} \bar{r}\right) s \theta(\beta) \lambda<v\left(x_{2} r\right) s<\bar{\lambda} v\left(x_{1} r\right) s$,

(2) $\lambda \theta(\beta) s v\left(x_{2} r-y_{2} \bar{r}\right)<s v\left(x_{2} r\right)<s \bar{\lambda} v\left(x_{1} r\right)$.

Since $x y^{-1} \in Y_{\gamma}$ and $x y^{-1} \equiv x_{1} r / y_{1} \bar{r} \bmod (\gamma)$, we have

$$
v\left(x_{1} r\right) \vee v\left(y_{1} \bar{r}\right) \leqq \sigma(\gamma) v\left(x_{1} r-y_{1} \bar{r}\right) .
$$

Use of the latter in (1) and (2) gives us that

(3) $\bar{\lambda} v\left(x_{1} r\right) s \leqq[\bar{\lambda} \sigma(\gamma)] v\left(x_{1} r-y_{1} \bar{r}\right) s<v\left(x_{1} r-y_{1} \bar{r}\right) s \lambda$,

(4) $s \bar{\lambda} v\left(x_{1} r\right) \leqq s[\bar{\lambda} \sigma(\gamma)] v\left(x_{1} r-y_{1} \bar{r}\right)<\lambda s v\left(x_{1} r-y_{1} \bar{r}\right)$.

Substitution of (3) and (4) in (1) and (2) and cancellation of $\lambda$ gives (i) and (ii) above. Thus 3.1, I-IV hold.

The results of the previous sections are collected and summarized in the next theorem and the first corollary which simplifies condition (c).

3.7 TheOREM. Consider an integral domain $A$ with a valuation $v: A \backslash\{0\}=A^{*} \rightarrow \Gamma$ (see 1.1) into a cancellative totally ordered semigroup $\Gamma$. (It is not assumed that either $A$ or $\Gamma$ is commutative or that $1 \in A$.) Let $P \subseteq \Gamma$ be the subsemigroup of strict twosided increasers. Suppose the following hold.

(a) For any $\lambda_{1}, \lambda_{2} \in \Gamma$ there is a $\gamma \in P$ such that $\lambda_{2}<\gamma \lambda_{1}$.

(b) For $\gamma \in P$, the semigroups $S_{\gamma}=A^{*} / q(\gamma)$ (see 1.7) satisfies the right Ore condition that $x S_{\gamma} \cap y S_{\gamma} \neq \varnothing$ for each $x, y \in S_{\gamma}$ and also that $S_{\gamma}$ is cancellative on both sides.

If (a) and (b) hold then $A^{*}$ can be embedded in a group $G$. Now in addition to (a) and (b) assume that

(c) (i) there is a subset $P_{1} \subseteq P$ and functions $\theta: P_{1} \rightarrow P, \sigma: P_{1} \rightarrow P$ such that

$$
\sigma(\beta) \triangleleft \theta(\beta)<\theta(\beta)^{2} \leqq \beta
$$

for all $\beta \in P_{1}$ (for the definition of " $\triangleleft$ " see 1.11 ).

(ii) For any $\eta \in \Gamma$, there exists an $\alpha \in P$ with $\eta<\sigma(\alpha)$.

Then $A$ can be embedded in the division ring $A \subset G \cup\{0\}$. 
3.8 Corollary I. Assume (a), (b), and $\left(\mathrm{c}^{\prime}\right)$ :

(c') $P \neq \varnothing$ and each $\alpha \in P$ satisfies $\alpha \triangleleft \alpha^{2}$ (see 1.11). Then $P_{1}=\left\{\alpha^{4} \mid \alpha \in P\right\}$, $\theta\left(\alpha^{4}\right)=\alpha^{2}, \sigma\left(\alpha^{4}\right)=\alpha$ satisfy (c). Consequently $A \subset G \cup\{0\}$ is an embedding of $A$ in $a$ division ring.

3.9 COROLlaRY II. Under the assumptions (a), (b), and (c) of the theorem, suppose that $\Gamma$ also satisfies the right Ore condition that $\alpha \Gamma \cap \beta \Gamma \neq \varnothing$ for any $\alpha, \beta \in \Gamma$. Then $v$ has a unique, natural extension to a valuation $\bar{v}: G \cup\{0\} \rightarrow \bar{\Gamma}$, where $\bar{\Gamma}$ is the group of quotients of $\Gamma$.

Proof. If $\alpha / \beta, \gamma / \delta \in \bar{\Gamma}$ with $\alpha, \beta, \gamma$, and $\delta \in \Gamma$, then find $\rho, \bar{\rho} \in \Gamma$ with $\beta \rho=\delta \bar{\rho}$ $\in \beta \Gamma \cap \delta \Gamma$. Then the definition

$$
\alpha / \beta<\gamma / \delta \text { if and only if } \alpha \rho<\gamma \bar{\rho} \text { in } \Gamma
$$

makes $\bar{\Gamma}$ into a totally ordered group. For $x=\left\{x_{\alpha}\right\} \in G$, pick any $\beta \in \Gamma$, any $x_{1}$, $x_{2} \in A^{*}$ with $x_{\beta}=x_{1} / x_{2} \bar{q}(\beta)$. Then the definition $\bar{v}(x)=v\left(x_{1}\right) / v\left(x_{2}\right)$ is independent of both $\beta$ and $x_{1}, x_{2}$. In order to verify 1.1 (i)-(iii), take $y_{1}, y_{2} \in A^{*}$ with $y_{\beta}=$ $y_{1} / y_{2} \bar{q}(\beta)$. If $c, \bar{c} \in A^{*}$ with $x_{2} c \sim y_{1} \bar{c} \bmod (\beta)$, then

$$
\begin{aligned}
\bar{v}(x y) & =\bar{v}\left(x_{1} c / y_{2} \bar{c}\right)=v\left(x_{1}\right) v(c) / v\left(y_{2}\right) v(\bar{c}) \\
& =\left[v\left(x_{1}\right) / v\left(x_{2}\right)\right]\left[v\left(y_{1}\right) / v\left(y_{2}\right)\right]=\bar{v}(x) \bar{v}(y) .
\end{aligned}
$$

By 3.1, $\bar{v}(x-y)=\bar{v}\left[\left(x y^{-1}\right) \psi(-y)\right]$. Take $\beta>\eta\left(x y^{-1}\right)$. There exist $r, \bar{r} \in A^{*}$ with $x_{2} r \sim y_{2} \bar{r} \bmod (\beta)$ so that $\left(x y^{-1}\right)_{\beta} \equiv x_{1} r / y_{1} \bar{r} \bmod (\beta)$ and

$$
\bar{v}\left[\left(x y^{-1}\right) \psi(-y)\right]=\bar{v}\left[\left(x y^{-1}\right) \psi\right] \bar{v}(y)=v\left(y_{1} \bar{r}-x_{1} r\right) / v\left(y_{2} \bar{r}\right) .
$$

If $v\left(y_{1} \bar{r}-x_{1} r\right) \leqq v\left(y_{1} \bar{r}\right)$, then $\bar{v}(x-y) \leqq \bar{v}(y)$. If $v\left(y_{1} \bar{r}-x_{1} r\right) \leqq v\left(x_{1} r\right)$, then since $v\left(y_{2} \bar{r}\right)=v\left(x_{2} r\right)$, it follows that $\bar{v}(x-y) \leqq \bar{v}(x)$. Thus $\bar{v}$ satisfies 1.1 (i)-(iii).

The next definition and corollary give the main source of examples of rings $A$ which can be embedded in division rings.

3.10 Definition. Consider a totally ordered cancellative semigroup $\Gamma$ and any commutative field $R$ (not necessarily of characteristic zero). If $b: \Gamma \rightarrow R$ is any function, then the support of $b$ is the set $\operatorname{supp}(b)=\{s \in \Gamma \mid b(s) \neq 0\}$. The additive group of all functions $b$ such that supp $(b)$ satisfies the ascending chain condition will be denoted by $V=V(\Gamma, R)$. In particular, let $v(b)$ denote the maximal element of supp (b). If $a, b \in V$ then $a b \in V$ is defined at $s \in \Gamma$ by

$$
\begin{aligned}
(a b)(s)= & \sum a(t) b(u) \\
& (t, u), s=t u .
\end{aligned}
$$

It is known that the above sum for each $s$ is finite and also that supp (ab) satisfies the ascending chain condition. The subset $P(\Gamma, R)$ consisting of all $b \in V$ with supp $(b)$ finite is just the ordinary semigroup ring over $\Gamma$. Then $v$ is a valuation in the sense of 1.1 for both $V$ and $P(\Gamma, R)$. 
Note that any commutative totally ordered group satisfies (i)-(iii) in the next corollary.

3.11 CoRollary III. With $V=V(\Gamma, R)$ as above and for $P \subseteq \Gamma$ the subsemigroup of strict increasers, assume the following.

(i) For any $\lambda_{1}, \lambda_{2} \in \Gamma$, there is a $\gamma \in P$ with $\lambda_{2}<\gamma \lambda_{1}$.

(ii) For each $\gamma \in P, \gamma \triangleleft \gamma^{2}$ (see 1.11).

(iii) For any $\gamma, \lambda \in \Gamma$, there exists $t \in \Gamma$ such that $\gamma$ st $\leqq s \lambda$ and $t s \gamma \leqq \lambda$ s holds for all $s \in \Gamma^{1}$.

(iv) Suppose $A \subseteq V(\Gamma, R)$ is a subring having the property that for arbitrary $a, b \in A^{*}$ there exist $a_{1}, b_{1} \in A^{*}$ with $v\left(a_{1}\right), v\left(b_{1}\right) \in P$ such that $\left(a a_{1}\right)(s)=\left(b b_{1}\right)(s)$ holds for all $s \geqq t$ for any given $t \in \Gamma$.

(v) If $\alpha, \gamma, \beta, \bar{\alpha}, \bar{\beta} \in \Gamma^{1}$ satisfy $\alpha \gamma \beta \leqq \bar{\alpha} \gamma \bar{\beta}$, then $\alpha \beta \leqq \bar{\alpha} \bar{\beta}$.

Then $A$ can be embedded in a division ring.

Proof. Given $a, b \in A^{*}$ and $\gamma \in P$, it has to be shown that in our previous notation $\left(a a_{1}, b b_{1}\right) \in q(\gamma)$ for some $a_{1}, b_{1} \in A^{*}$. Take $\lambda=v(a)$ and let $t$ be as given by (iii). Then $a a_{1} \sim b b_{1} \bmod (\gamma)$, since for any $s \in \Gamma^{1}$, it follows from (iv) that

(i) $\gamma s v\left(a a_{1}-b b_{1}\right) \leqq \gamma s t \leqq s v(a)<s v\left(a a_{1}\right)$,

(ii) $v\left(a a_{1}-b b_{1}\right) s \gamma \leqq t s \gamma \leqq v(a) s<v\left(a a_{1}\right) s$.

Thus 3.8(a), (b), ( $\left.c^{\prime}\right)$ hold and $A$ can be embedded in a division ring.

3.12. Suppose $A$ is a ring with a valuation into the integers, i.e. $v: A \rightarrow \Gamma=Z$. Define $A_{n}=\{a \in A \mid v(a)=n\}$ and $B_{n}$ as the additive quotient group $B_{n}=A_{n}-A_{n-1}$. Form the associated graded algebra $G(A)=\oplus\left\{B_{n} \mid n=0, \pm 1, \ldots\right\}$ (see [5, p. 257]). Then Cohn has shown [2, p. 523, Theorem 4.2] that in this particular case the assumption 3.7(b), i.e. that for each $a, b \in A^{*}$, the function $f(x, y)=v(a x-b y)$ $-v(a x)$, with $x, y \in A^{*}$, is not bounded below is equivalent to requiring that the ring $G(A)$ satisfies the right Ore condition. If in the last corollary $\Gamma=Z$, then $A$ is a subring of the ordinary power series ring $V(\Gamma, R)$, while $G(A)=P(\Gamma, R)$, and condition 3.11 (iv) specializes to requiring that $P(\Gamma, R)$ satisfies the Ore condition.

In conclusion, some totally ordered semigroups are described which could be used for a valuation and which are also useful in illustrating the relation " $\triangleleft$ " (see 1.11). Various subsemigroups of wreath products of totally ordered groups are too well known and will not be mentioned.

3.13 EXAMPLE. Let $\cdots<x_{2}^{-1}<x_{1}^{-1}<e=1<x_{1}<x_{2}<\cdots$ be any finite or infinite totally ordered set indexed by ordinals. Let $\Gamma$ be the group generated by this set, i.e. all expressions $z=z_{1} z_{2} \cdots z_{m}$, where $m$ is an integer and where each $z_{i}=x_{j}$ or $x_{j}^{-1}$ and $x_{j} x_{j}^{-1}=x_{j}^{-1} x_{j}=e$. If $p(z)$ denotes the total number of $z_{i}$ with positive and $n(z)$ the number with negative exponents, set $\partial(z)=p(z)-n(z)$. Note that $\partial(e)=p(e)=n(e)=0$ and $\partial\left(x_{j}^{k}\right)=k$ for any positive or negative integer $k$. If $u=u_{1} u_{2}$ $\cdots u_{r} \in \Gamma$ is another element (where $r$ is an integer and $u_{i}=x_{j}$ or $x_{j}^{-1}$ ), define $z<u$ if $\partial z<\partial u$; or $\partial z=\partial u$, but $z_{t}<u_{t}$ for the first $t$ with $z_{t} \neq u_{t}$. Then $\Gamma$ is a totally ordered group, $P=\{z \in \Gamma \mid 1 \leqq \partial z\}$, and $z \triangleleft u$ if and only if $\partial z<\partial u$. 
3.14 ExAmple. Consider again a totally ordered set $1=e<x_{1}<x_{2}<\cdots$ indexed by ordinals. Let $\Gamma$ consist of $e$ and the set of all finite or infinite formal expressions $z$ of the form $z=z_{1} z_{2} \cdots z_{\alpha}$, where each $z_{i}$ equals some $x_{j}$, and where the $z_{i}$ are indexed by ordinals in increasing order, and where $\alpha$ is the biggest index. Then $\Gamma$ is a semigroup with juxtaposition as multiplication. If $u=u_{1} u_{2} \cdots u_{\beta} \in \Gamma$, where $u_{i}$ equals some $x_{j}$, then $z u=z_{1} z_{2} \cdots z_{\alpha} t_{\alpha+1} \cdots t_{\alpha+\beta}$ where $t_{\alpha+1}=u_{1}$ and $t_{\alpha+\gamma}=u_{\gamma}$. The degree $\partial z$ of $z$ is $\partial z=\alpha$. Define $z<u$ if either $\partial z<\partial u$, or if $\partial z=\partial u$, but $z_{t}<u_{t}$ for the first ordinal $t$ such that $z_{t} \neq u_{t}$. Then $\Gamma$ is a totally ordered cancellative semigroup where $z \triangleleft u$ if and only if $\partial z<\partial u$.

\section{REFERENCES}

1. A. J. Bowtell, On a question of Mal'cev, J. Algebra 7 (1967), 126-139. MR 37 \#6310.

2. P. M. Cohn, On the embedding of rings in skew fields, Proc. London Math. Soc. (3) 11 (1961), 511-530. MR 25 \#100.

3. P. Conrad, and J. Dauns, An embedding theorem for lattice-ordered fields, Pacific J. Math. 30 (1969), 385-398.

4. L. Fuchs, Partially ordered algebraic systems, Pergamon Press, Oxford and AddisonWesley, Reading, Mass., 1963. MR 30 \#2090.

5. N. Jacobson, Structure of rings, rev. ed., Amer. Math. Soc. Colloq. Publ., vol. 37, Amer. Math. Soc., Providence, R. I., 1964. MR 36 \#5158.

6. A. A. Klein, Rings nonembeddable in fields with multiplicative semigroups embeddable in groups, J. Algebra 7 (1967), 100-125. MR 37 \#6309.

TULANE UNIVERSITY,

New OrLeans, Louisiana 70118 